\title{
Protein Characteristic for New Beginners
}

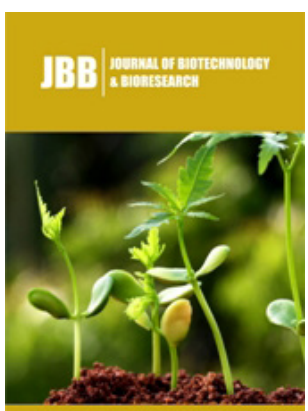

*Corresponding author: Lay-Harn Gam, School of Pharmaceutical Sciences, Universiti Sains Malaysia, 11800 Penang, Malaysia

Submission: 侮July 11, 2019

Published: 酶July 17, 2019

Volume 2 - Issue 2

How to cite this article: Lay-Harn Gam. Protein Characteristic for New Beginners. J Biotech Biores.2(2). JBB.000533.2019.

Copyright@ Lay-Harn Gam, This article is distributed under the terms of the Creative Commons Attribution 4.0 International License, which permits unrestricted use and redistribution provided that the original author and source are credited.

\author{
Lay-Harn Gam* \\ School of Pharmaceutical Sciences, Malaysia
}

\section{Opinion}

One of the branches of biotechnology is proteomics. It is an interesting field as proteins, unlike DNA which made up of combination of 4 nucleotides, is made up of combination of 22 amino acids, where the sequence of the amino acids gives uniqueness to each protein. In addition, the structure of the protein such as primary, secondary, tertiary and the folding of protein into quaternary making the protein different although they may have similar amino acid sequence. Furthermore, a functional protein may be consisting of more than one polypeptide. Each of the amino acid has different characteristic therefore the combination of the amino acids making protein to have different degrees of polarity. In addition, the post translation modification of protein, such as glycosylation, lipid components and metal ions making the protein to function differently according to their activity. All this made handling of protein difficult and if one is not careful, it may cause the problem in reproducibility of protein based experimental work.

In the field of proteomics bioanalysis, solubility of protein is upmost important as one can only analyze the protein when it is solubilized. Increase surface area will result in high yield of protein extract, this can be done by homogenizing the protein source sample (tissue, cell and etc.). In order to reduce the complex protein mixture, one can extract protein into two mixtures, name aqueous soluble mixture and membrane bound protein mixture. Aqueous soluble proteins can be extracted using buffer solution, However, in order to solubilize hydrophobic proteins, which are normally membrane bound proteins, combination of different reagents can be used.

Separation of proteins by gel electrophoresis is a common and robust method in protein analysis. In one dimensional gel electrophoresis, sodium dodecyl sulphate is usually added to give an overall negative charge to the proteins and therefore separation is based solely on the molecular weight of proteins. However, the resolution of one-dimensional gel electrophoresis is relatively poor, which resulted in overlapping of different kind of proteins. If one is to separate complex mixture of protein, it is best of consider two dimensional gel electrophoresis, where proteins are separated according to their isoelectric point and molecular weight, however one have to be careful not to add any chemicals that can cause the change of isoelectric point of protein in sample preparation. Protein samples to be separated using two-dimensional gel electrophoresis has to be cleaned thoroughly in order to obtain good protein separation.

When activity is of concerned, protein must be handled with care, the extraction work is usually done at low temperature and the use of stringent chemicals should be avoided, this is because temperature can denature proteins folding while chemicals can change the ionic strength of the protein and causing denaturation of protein. Storage of protein is to be done in small aliquate and best to store protein at $-80^{\circ}$, this is because slow freezing of protein can cause protein denaturation.

The field of proteomics is fast advancing. It is a useful tool in biopharmaceuticals and bioresearch and subsequently benefiting mankind. Nevertheless, one should understand the protein characteristic in order not to misinterpreting the research outcome.

For possible submissions Click below: 